\title{
Resection and Reconstruction of Acetabular Tumors involving Ischium and Inferior pubic bone with a Total Hip Replacement Using Posterior K-L Approach
}

DONGQING ZUO ( $\nabla$ dongqing.zuo1@shgh.cn )

Shanghai General Hospital Department of Orthopaedics: Shanghai Jiaotong University First People's Hospital Department of Orthopaedics

Mingyang Yu

Affiliated Zhongshan Hospital of Dalian University

Haoran Mu

Shanghai General Hospital

Mengxiong Sun

Shanghai General Hospital

Jiakang Shen

Shanghai General Hospital

Chongren Wang

Shanghai General Hospital

Hongsheng Wang

Shanghai General Hospital

Xiaojun Ma

Shanghai General Hospital

Qi Ruan

Shanghai General Hospital

Yu Lv

Shanghai General Hospital

Wei Sun

Shanghai General Hospital

Zhengdong Cai

Shanghai General Hospital

\section{Research Article}

Keywords: Pelvic tumor, Kocher langenbeek approach, Pelvic zone III 
Posted Date: August 23rd, 2021

DOl: https://doi.org/10.21203/rs.3.rs-805300/v1

License: (c) (i) This work is licensed under a Creative Commons Attribution 4.0 International License. Read Full License 


\section{Abstract}

Background: To explore the feasibility and short-term effect of posterior K-L (Kocher Langenbeek) approach in treating pelvic acetabular tumors involving ishium, inferior ramus of pubis.

Methods: We retrospectively reviewed patients with acetabular tumors involving ishium, inferior ramus of pubis underwent surgery in Shanghai General Hospital from January 2016 to June 2020 with modified K$\mathrm{L}$ approach, including 8 women and 12 men, patients aged from 19-76 years, including 6 chondrosarcomas, 6 osteosarcomas, 3 metastatic pelvic tumors, 2 epithelioid sarcomas, 3 giant cell tumor of bone. 20 patients were defined as pelvic Ennecking zone II + pelvic zone III involvement. All patients were followed up regularly in clinic, operation time, intraoperative bleeding, methods of reconstruction, duration of hospitalization, perioperative complications were recorded, and MSTS functional score was used to evaluate the patients' functional status.

Results: All patients were followed up regularly in clinic with a mean 32 months (20-48 months). No patient died in the perioperative period, the average intraoperative bleeding was $1250 \mathrm{ml}(800-2400 \mathrm{ml})$ in 10 patients, the mean operation duration was 2.6 hours (2-3.5 hours), no wound infection and delayed healing, the average duration of hospital stay was 12 days (10-16 days), the main complications during the follow-up included nervus cutaneus femoris posterior paralysis, 2 tumor recurrence, MSTS score was 22.5 points. 4 patients died during follow-up.

Conclusions: The posterior K-L approach can be employed to effectively resect and reconstruction pelvic acetabular tumors invoving ishium, inferior ramus of pubis and reconstruct them properly. It is mainly suitable for malignant pelvic tumors involving the pelvic III and II + III regions. It also can often be available for biological reconstruction by using the acetabulum tantalum metal block, acetabulum reinforcing ring or autograft femur head. It is of highly safety and few perioperative complications, which is worth popularizing.

\section{Background}

The pelvic ring is composed of iliac bone, ischium, pubis and part of sacral symphysis. It is the prone site of a variety of primary (chondrosarcoma, Ewing sarcoma and osteosarcoma) and metastatic tumors in the axial bone of the human body [1]. The classic Enneking pelvic tumor division divides the pelvis into four regions: the iliac bone is region I, acetabulum is zone II, the ischium and pubis are zone III, acral wing is zone IV. Due to the complexity of anatomy and biomechanics of pelvic ring, the resection and reconstruction of pelvic tumors involving pelvic ring are more difficult than those of limbs. Some pelvic tumors can involve the ischium, pubis and acetabulum at the same time. The resection and reconstruction of such pelvic tumors are the most challenging, involving how to select the surgical approach for complete tumor resection and how to carry out functional reconstruction. At present, there is still a lack of unified treatment standards, which is a hot issue in pelvic surgery [2]. 
The classic surgical approaches for pelvic tumors include Smith Peterson approach and ilioinguinal approach. Most of the pelvic tumor surgey including hemipelvic resection and replacement can be achieved. However, some pelvic tumors involving the acetabulum are difficult to expose during the operation due to the deep anatomical position of the sciatic and pubic branches and the surrounding attached muscles and ligaments. At present, there is no ideal surgical approach. Ilioinguinal approach combined with medial thigh incision is often used to remove such tumors. The surgical method of removing tumors of the lower pubic branch through the medial thigh perineal approach alone can effectively target the tumors of the lower pubic branch, separate the muscles by using the muscle space, and directly reach the lower pubic branch, which can effectively avoid the important nerves and blood vessels of the pelvis. The K-L approach (Kocher langenbeek) is a classic approach for the treatment of acetabular fractures and joint surgery [3], which can directly expose the posterior column of the acetabulum and look directly at the upper wall and quadrilateral of the acetabulum. At present, no scholars have reported the resection of pelvic tumors through K-L related approach, especially the acetabular tumors with accumulated sciatic and inferior pubic branches.

Resection of pubic and sciatic tumors in Zone III has little impact on the stability of the pelvic ring, and stability reconstruction is often not required. However, when pubic and sciatic tumors involve the acetabulum, hip reconstruction is required after complete tumor resection. Hemipelvic replacement is one of the standard treatments for partial pelvic tumors involving acetabulum, but the incidence of wound complications, prosthesis dislocation and fracture is high, and the limb function score is relatively poor. Clinical studies show that the long-term prosthesis survival rate and limb function of biological reconstruction are significantly better than that of abiotic reconstruction, which is suitable for tumor patients with better life expectancy.

This study retrospectively analyzed the 20 cases of acetabular tumors involving the sciatic and inferior pubic branches, analyzed its clinical safety and feasibility, and followed up the short-term postoperative results. We then proposed posterior resection method for acetabular tumors involving the sciatic and inferior pubic branches, that is, the acetabular tumors involving the sciatic and inferior pubic branches were removed through single K-L approach (Kocher langenbeek), and the acetabulum was reconstructed through autologous femoral head, tantalum block and porous acetabular cup.

\section{Methods}

\section{Participants}

From January 2016 to June 2020, a total of 33 patients with pelvic ischium and inferior pubic branch involving acetabular tumors were reviewed from the department of bone oncology of Shanghai General Hospital Affiliated to Shanghai Jiaotong University. 20 patients were included in this study. There were 12 males and 8 females; The age ranged from 19 to 76 years, with an average of 45.8 years; It included 6 cases of chondrosarcoma, 6 cases of osteosarcoma, 2 cases of epithelioid sarcoma, 1 case of renal cancer metastasis, 1 case of lung cancer metastasis, 1 case of liver cancer metastasis and 3 cases of 
giant cell tumor of bone; All 20 patients involved the hip joint, which was divided into zone II + III according to Enneking pelvic tumor division.

\section{Preoperative Management}

20 patients in this group underwent biopsy before operation to confirm pathology. The main symptoms before operation were local pain. there were 10 cases of osteolytic destruction, osteogenic destruction in 6 cases and mixed bone destruction in 4 cases. All patients underwent X-ray, CT scan and MRI before operation, and PET-CT scan to determine the general condition and metastatic status. This study has been approved by the ethics committee of our hospital, and all patients signed informed consent.

All patients underwent preoperative internal iliac angiography to determine the tumor blood supply and tumor vascular embolization within 1-2 days before operation. According to the blood supply of internal iliac artery branches, gelatin sponge embolization was routinely used during operation. Patients with giant cell tumor of bone should use desudumab (120 Mg, 3-4 times) before operation, the patients with abundant blood supply can be blocked by abdominal aortic balloon or ligated by internal iliac artery during operation. The pelvic bone and soft tissue involvement was evaluated by enhanced CT and magnetic resonance images, and the osteotomy during operation was determined. The acetabular defect was often repaired with appropriate tantalum metal patch or autologous femoral head patch combined with cannulated screw fixation.

\section{Tumor Resection And Reconstruction}

In brief, general anesthesia was applied to all patients, patients were then placed with lateral position, slightly pading up the affected lower limb, and maintain the neutral position of the lower limb. During the operation, the affected limb can bend the hip and knee, adduct and rotate the lower limb, and the pelvis is perpendicular to the operating table. The affected lower limbs were routinely disinfected, and the proximal end was disinfected to a $15 \mathrm{~cm}$ area near the proximal end of the iliac spine. The skin incision is shown in Fig. 1. The incision passes through the posterior lower corner of greater trochanter to the area between sciatic nodules, with a length of about $20-25 \mathrm{~cm}$. The osteotomy plan was determined through intraoperative fluoroscopy combined with preoperative CT and MRI reconstruction. For patients with difficulty in osteotomy, the tumor was completely removed through intraoperative o-arm combined with intraoperative navigation and positioning.

For patients involving acetabulum, the acetabular bone defect was reconstructed individually according to the acetabular bone defect. Our previous clinical research summarized our experience in acetabular defect reconstruction [4]. According to the classification of acetabular defect, it can be classified as three types: type A: the tumor involves the anterior acetabular edge, after tumor resection, the posterior acetabular column has complete continuity and good structural bone graft stability; Type B: the tumor of sciatic branch involved the posterior lower part of acetabulum. After tumor resection, the continuity of 
acetabular anterior column was complete and the stability of structural bone graft was poor; Type C: iliac bone tumor involves the upper edge of acetabulum. After tumor resection, pelvic continuity is interrupted. There are many bone defects above acetabulum, which require autologous bone grafting and large bone volume. This group of cases belong to pelvic continuity, and some acetabular defects. The classification is type B defect after acetabular resection [5]. The key points of acetabular reconstruction are mainly the defect at the posterior edge of acetabulum after tumor resection, which is difficult to do structural bone grafting and fixation. Therefore, after tumor resection and osteotomy, the acetabular repair method can be determined according to the defect size, tumor type and expected survival. For example, acetabular reinforcing ring and tantalum metal reconstruction are often used for metastatic tumors with short expected survival time; In case of low-grade malignancy or young age, autologous femoral head bone block is preferred, and the autologous bone is fixed above the acetabulum by cannulated screw. The specific operations are as follows: firstly, grind the acetabulum, choose proper method according to the size of the defect, fix and install the acetabular reinforcing ring with the front residual bone, then fix the bone graft block on the front residual bone and acetabular reinforcing ring, trim the excess bone into granules after installation, implant into the structural bone graft, and then install the acetabulum; If it is an autologous femoral head, first fix the femoral head with acetabular screws, and then grind and file the acetabulum. After installing the acetabular prosthesis, decide whether to use tantalum block for fixation according to its stability, as shown in Fig. 2 (partial acetabular osteotomy, reconstruction of femoral head and tantalum block). The position and rotation center of the prosthesis are determined according to the residual acetabulum. At the same time, the femoral anteversion angle is adjusted to increase the joint stability. During the operation, the polyethylene padded acetabular prosthesis with high edge is used to reduce the risk of posterior dislocation, and the large femoral head is used as much as possible to increase the joint stability. The stability of the hip joint was tested by completely suturing the external rotator muscle, repairing the gluteus medius and gluteus maximus, and repairing the joint capsule.

\section{Postoperative Management And Follow-up}

Negative pressure drainage is routinely placed after operation. The second-generation cephalosporin is used for 2-3 days after operation. The drainage tube is removed according to the postoperative drainage flow. If the drainage flow is less than $50 \mathrm{ml}$ within 24 hours after operation, the drainage tube is removed for no more than 3 days in principle, so as to prevent retrograde infection of the drainage tube. One week after operation, the patient was instructed to sit up at the bedside, the wound healed well after operation, and early ground activities were encouraged. The patients who underwent partial acetabular resection and reconstruction were helped to stand with partial weight-bearing of double crutches, accompanied with progressive lower limb muscle function exercise, and fixed with lower limb anti rotation shoes for 4 weeks during bed rest.

Pelvic X-ray examination was performed after drainage tube was removed. The patients were followed up at 6 weeks, 3 months and 6 months after operation. The pelvic CT, X-ray film and lung CT were rechecked. 
Bone scan was performed once every half a year to evaluate the local control of the tumor; The affected limb function was evaluated by msts-93 score of Musculoskeletal Tumor Society [6].

\section{Results}

There was no death during the perioperative period, and the patients recovered and discharged after operation (Fig. 3 and Fig. 4 are the diagnosis and treatment information of typical patients). All 20 patients underwent partial acetabular + zone III resection and reconstruction with autologous femoral head and tantalum metal patch; The operation time was 2-3.5 hours, with an average of 2.6 hours; Intraoperative bleeding was $800-2400 \mathrm{ml}$, with an average of $1250 \mathrm{ml}$; The hospital stay was 10-16 days, with an average of 12 days. Two patients with giant cell tumor of bone were treated with denosumab injection four times before operation, one patient with metastatic tumor of renal cell carcinoma underwent internal iliac artery ligation during operation, and the other 17 patients underwent preoperative internal iliac artery angiography and gelatin sponge embolization. The primary wound healing rate was $100 \%$.

20 patients were followed up for 20-48 months, with an average of 32 months. During the follow-up period, 4 patients died, 2 patients recurred after operation, 1 patient began to follow up irregularly one year after operation due to the progression of the primary disease, and the patient died one and a half years after operation. The three-year overall survival rate of this group was 75\% (Fig. 5). 15 patients had lesions involving acetabulum, ischium and inferior pubic branch, and 5 patients had lesions of acetabulum and partial ischium.

Of the 6 patients with osteosarcoma, 2 had recurrence. 1 cases of osteosarcoma had a history of breast cancer. Preoperative PET-CT and puncture were all indicated as metastatic tumors of II + III breast cancer. Tumor resection plus acetabular reinforcement ring total hip arthroplasty, postoperative pathology showed osteosarcoma, postoperative adjuvant chemotherapy was refused, local recurrence occurred half a year after operation, and proton heavy ion treatment was carried out in the external hospital. Another patient with osteosarcoma underwent sciatic tumor resection + hip arthroplasty three years ago. The postoperative pathology was osteosarcoma. The patient refused postoperative adjuvant chemotherapy for personal reasons, and there was no regular follow-up during the period. Three years after operation, it recurred in situ, and then underwent anterior tumor resection + hemipelvic replacement. The postoperative follow-up was generally acceptable. There were 3 cases of metastatic tumors, 1 case of lung cancer was treated with lung cancer targeted drugs, and 1 case of renal cancer was treated with sotan after operation. The tumor drug resistance progressed and died 20 months after operation. Patients with liver cancer metastasis had no adjuvant treatment, and patients with renal cancer and liver cancer died 30 months after operation. Patients with giant cell tumor of bone underwent subcutaneous injection of denosumab for 6 months. Six patients with chondrosarcoma were followed up without radiotherapy and chemotherapy. There was no local recurrence and metastasis during the follow-up. 
Functional evaluation: the functional score was evaluated according to the results of the last follow-up. According to the six aspects of pain, limb function, satisfaction, brace assistance, walking and gait during the follow-up, msts-93 score was $21 \sim 26$, with an average of 22.5, of which 14 cases were excellent and 6 cases were good. During the follow-up period, 20 patients had no postoperative complications such as joint dislocation and wound nonunion. The average time of VSD negative pressure suction in 20 patients was 5 days. Wound complications such as perioperative wound infection and flap necrosis occurred. 2 patients had numbness in the rear of hip and thigh after operation. They were followed up for 6 months. There were no symptoms of sciatic nerve injury.

\section{Discussion}

\section{Efficacy analysis and indications of K-L approach and reconstruction}

Compared with the classical K-L approach, the modified K-L approach in our study can be more fully exposed to the sciatic tubercle, the distal femur to the far end of the trochanter, and the sciatic branches and acetabulum can be exposed directly after flipping the gluteus maximus myocutaneous flap. The sciatic tumor can be completely removed during the operation. If the acetabulum is involved, the acetabulum can be exposed and the tumor can be removed. Therefore, the posterior K-L approach is mainly applicable to the resection of pelvic tumors in pelvic region III sciatic bone, part of inferior pubic branch and part of type II + III tumors, mainly low-grade malignant tumors or progressive benign lesions, and some proximal femoral tumors are also applicable. This study found that it has good short-term results, high safety and less perioperative complications, which is worthy of promotion. This approach can provide sufficient joint exposure and is suitable for hip replacement, hip reconstruction with reinforcing ring and other biological reconstruction [5]. Reconstruction of acetabular defect with artificial metal hemipelvic prosthesis is a classic reconstruction method involving acetabular tumors, but the longterm survival rate of non-biological reconstruction prosthesis is still low, prosthesis related complications are high, and the function of affected limbs is poor [7-9]. Previous literature reviewed a number of clinical studies $[9,10]$ and compared the clinical prognosis and function of patients after different reconstruction methods. The data showed that the average MSTS after hip displacement and open placement after hemipelectomy was 20.8 points, while the saddle prosthesis was only 16 points, and the customized modular hemipelvic prosthesis was 18.6 points. The best postoperative function was autologous hemipelvic radiation inactivation reconstruction, the average MSTS score was 23 . Our department reported the follow-up results of this prosthesis + tantalum block / autologous femoral head reconstruction method in 2019 [11], 20 patients were followed up for an average of 48 months. During the follow-up, 2 cases recurred, 1 case had prosthesis loosening, 13 patients had excellent MSTS function score, 6 cases were good and 1 case was poor. The follow-up results were satisfactory. In this study, there were no major complications such as joint dislocation, deep wound infection and dislocation. The average intraoperative bleeding was about $1250 \mathrm{ml}$, which was lower than that of patients undergoing hemipelvic replacement at the same time. The average postoperative functional score was 22.5 , which 
was better than that of patients undergoing hemipelvic resection and replacement, hip joint exclusion and hemipelvic inactivation. All tumor patients in this group are feasible for biological reconstruction. The joint prosthesis is stable and the soft tissue repair is good. The prosthesis stability is good during postoperative follow-up. No patient has postoperative joint dislocation, prosthesis loosening and fracture. The joint stability is significantly better than that of patients who underwent hemipelvic replacement at the same time (data not published by the center). Most patients can walk with partial weight about one month after operation, the postoperative time was significantly shorter than that in patients with hemipelvic replacement and open hip joint.

\section{Limitations Of This Study}

The K-L approach used in this study often requires two major osteotomies for resection. Partial acetabular osteotomy can be performed under direct vision after fully exposing the acetabulum, while medial osteotomy is usually performed through the pubic symphysis; Occasionally, it is necessary to extend the approach to the contralateral pubic branch for limited type III resection. The medial osteotomy is deep, there are many pubic and sciatic muscles attached, and it is close to the urethra and bladder, and osteotomy can't be performed under direct vision. There may be osteotomy error and insufficient resection boundary of soft tissue tumors. This potential risk can increase the accuracy of osteotomy and reduce the possibility of residual tumor dissemination through preoperative accurate planning and intraoperative navigation. The author believes that this approach is not recommended for highly malignant primary bone tumors or cases involving bone destruction with obvious soft tissue involvement. In addition, this study is a retrospective case study without control group and randomized study setting. Secondly, there are relatively few cases in this study, the follow-up time is short, and the long-term effect of prosthesis reconstruction cannot be detected. In the future, it is necessary to prospectively study the shortcomings of different surgical approaches and reconstruction methods, so as to further improve and standardize the resection and reconstruction of this kind of pelvic tumor.

In conclusion, K-L approach can be used for resection and joint biological reconstruction of acetabular tumors of cumulative sciatic and inferior pubic branches. The operation has advantages of safe operation, good wound healing, stable joint and satisfactory short-term tumor follow-up results, which is worthy of popularization and application. In view of the small number of cases and short follow-up time, the tumor prognosis and long-term stability of prosthesis still need to be followed up.

\section{Abbreviations}

K- L

Kocher Langenbeek; CT:Computed Tomography; MRI:Magnetic Resonance Imaging; PET-CT:Positron Emission Tomography/Computed Tomography; VSD:Vacuum Sealing Drainage

\section{Declarations}




\section{Ethics approval and consent to participate}

This study was approved by the ethics committee of Shanghai General Hospital.

All patients signed informed consent.

\section{Consent for publication}

The patient was given his consent for information about himself to be published in World Journal of Surgical Oncology.

\section{Availability of data and materials}

The data used to support the findings of this study are available from the corresponding author upon request.

\section{Competing interests}

The authors declare that they have no competing interests.

\section{Funding}

National Natural Science Foundation of China $(81802985,81772859)$

\section{Authors' contributions}

DZ, MY, and WS conceived the study. MH performed the literature search and MS writing of the manuscript. JS analyzed and interpreted the data. CW, HW, and XM collected and assembled the data. $\mathrm{QR}, \mathrm{YL}, \mathrm{ZC}$ submitted the manuscript and is the corresponding author. All authors read and approved the final manuscript.

\section{Acknowledgements}

Not applicable.

\section{Author information}

Dongqing Zuo and Mingyang Yu are co-first authors.

\section{References}

1. Garcia JG, Martinez A, Filho RJG, Petrilli MT, Viola DC. Epidemiological characteristics of patients with pelvic tumors submitted to surgical treatment. Rev Bras Ortop. 2017 Dec;6(1):33-7. 53(.

2. Brown TS, Salib CG, Rose PS, Sim FH, Lewallen DG, Abdel MP. Reconstruction of the hip after resection of periacetabular oncological lesions: a systematic review. Bone Joint J. 2018 Jan;100- 
$B(1$ Supple A):22-30.

3. Magu NK, Rohilla R, Singh A, Wadhwani J. Modified Kocher-Langenbeck approach in combined surgical exposures for acetabular fractures management. Indian J Orthop. 2016 MarApr;50(2):206-12.

4. Mayerson JL, Wooldridge AN, Scharschmidt TJ. Pelvic resection: current concepts[J]. JAAOS-Journal of the American Academy of Orthopaedic Surgeons. 2014;22(4):214-22.

5. Sun W, Zuo DQ, Zan PF, Shen JK, Sun MX, Ze-Ze FU,ZD Cai. Feasibility and early efficacy of tantalum patch in the reconstruction of acetabular defect after resection of acetabular pelvic tumor. Chinese Journal of Bone and Joint, July 2019, Vol 8, No.7.

6. Enneking WF, Dunham W, Gebhardt MC, Malawar M, Pritchard DJ. A system for the functional evaluation of reconstructive procedures after surgical treatment of tumors of the musculoskeletal system. Clin Orthop Relat Res. 1993 Jan;(286):241-6. PMID: 8425352.

7. Ippolito J, Thomson J, Beebe K, Patterson F, Benevenia J. Outcomes following periacetabular tumor resection: A 25-year institutional experience. J Surg Oncol. 2020 Oct;122(5):949-54.

8. Erol B, Sofulu O, Sirin E, Saglam F, Buyuktopcu O. Reconstruction after periacetabular tumor resection with Lumic endoprosthesis: What are the midterm results? J Surg Oncol. 2021 Feb;123(2):532-43.

9. Xu H, Li Y, Zhang Q, Hao L, Yu F, Niu X. Does Adding Femoral Lengthening at the Time of Rotation Hip Transposition After Periacetabular Tumor Resection Allow for Restoration of Limb Length and Function? Interim Results of a Modified Hip Transposition Procedure. Clin Orthop Relat Res. 2021 Jul 1;479(7):1521-1530.

10. Gebert C, Wessling M, Hoffmann C, Roedl R, Winkelmann W, Gosheger G, Hardes J. Hip transposition as a limb salvage procedure following the resection of periacetabular tumors. J Surg Oncol. 2011 Mar 1;103(3):269 - 75.

11. Sun W, Zan P, Ma X, Hua Y, Shen J, Cai Z. Surgical resection and reconstructive techniques using autologous femoral head bone-grafting in treating partial acetabular defects arising from primary pelvic malignant tumors. BMC Cancer. 2019 Oct;18(1):969. 19(.

\section{Figures}




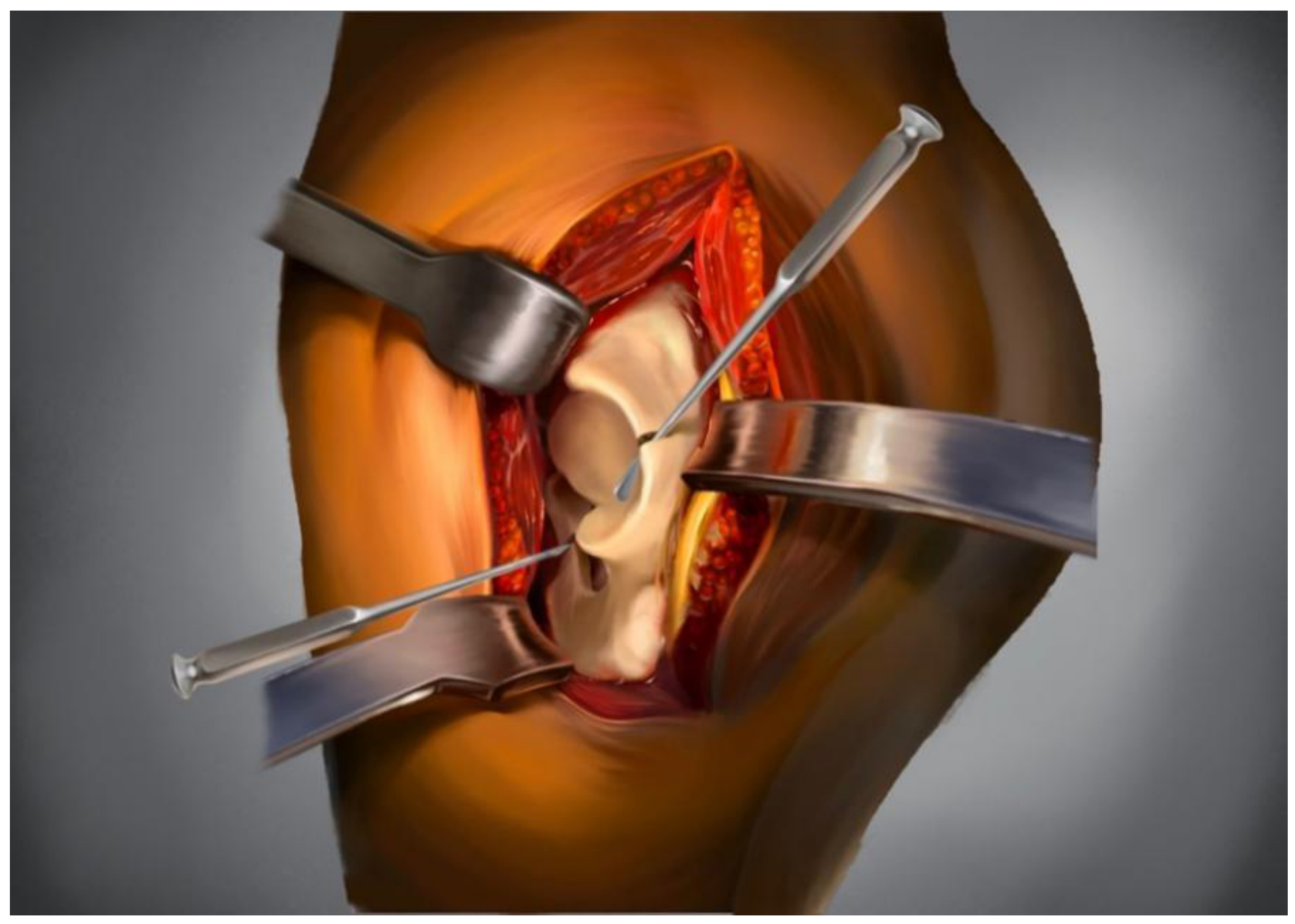

\section{Figure 1}

Schematic diagram of incision exposure and osteotomy planning of modified K-L approach. 


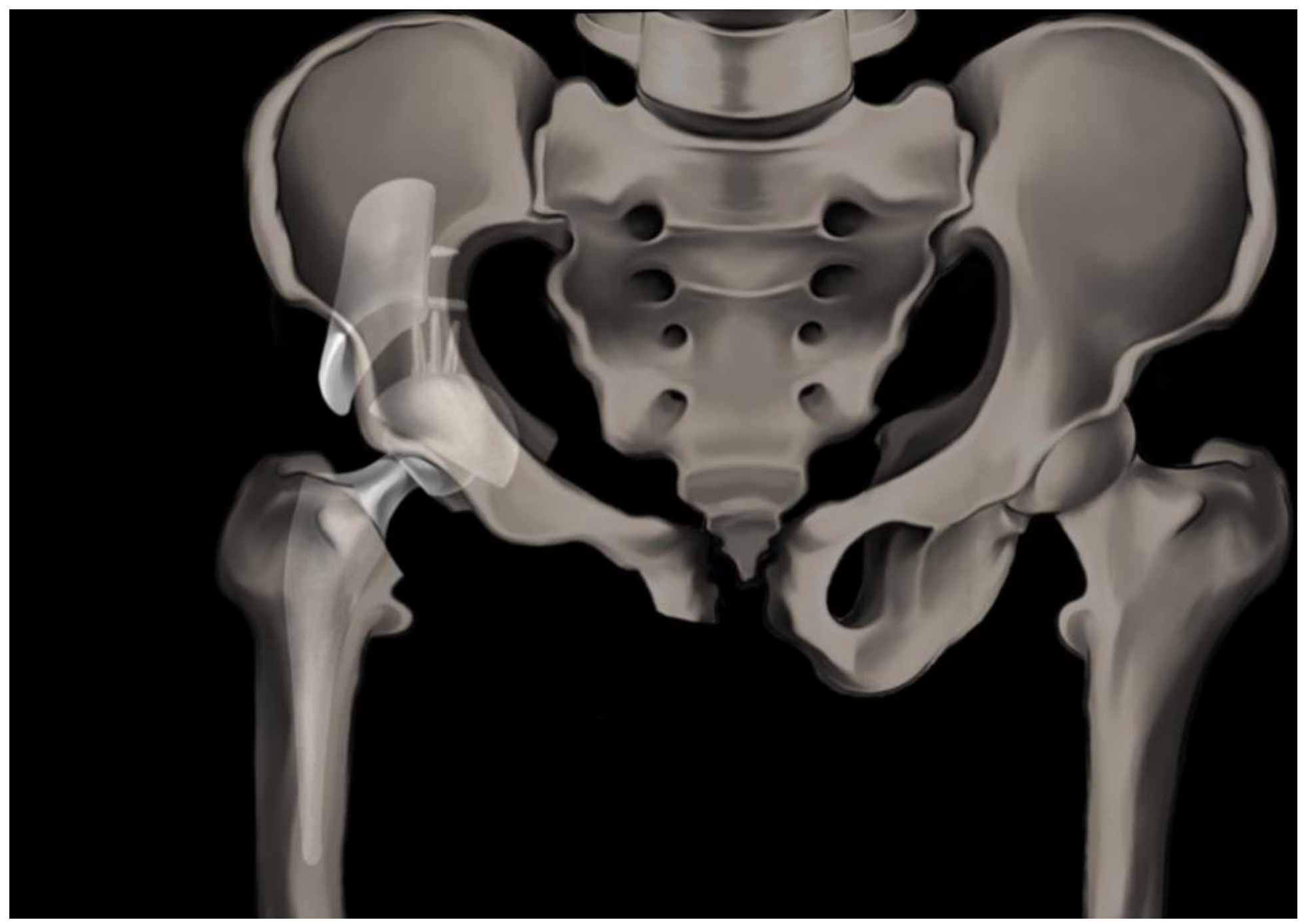

Figure 2

Schematic diagram of reconstruction after acetabular osteotomy defect. 

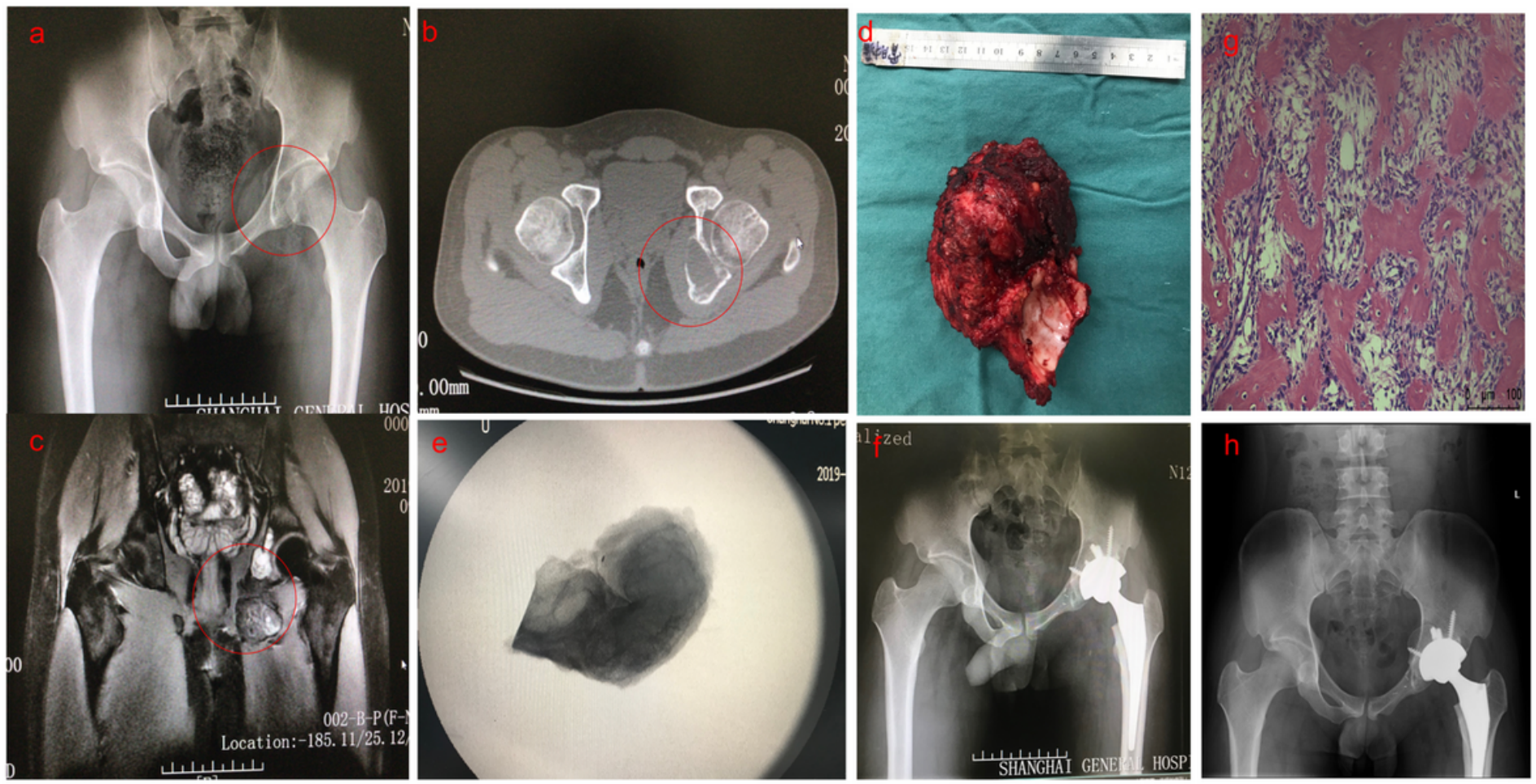

\section{Figure 3}

Male, 27 years old, GCTB in pelvic area II + III (a) Preoperative pelvic plain film showed bone destruction of the left acetabulum (b) Preoperative axial CT showed osteolytic destruction of the left pelvic ischium and acetabulum (c) Preoperative coronal MRI T1 showed bone destruction of the left acetabulum (d) Intraoperative gross specimens (e) Gross specimen fluoroscopy (f) One month after operation, the pelvic plain film showed that the joint was in place and the left ischium was absent (g) He sections of postoperative specimens (h) 24 months after operation. 

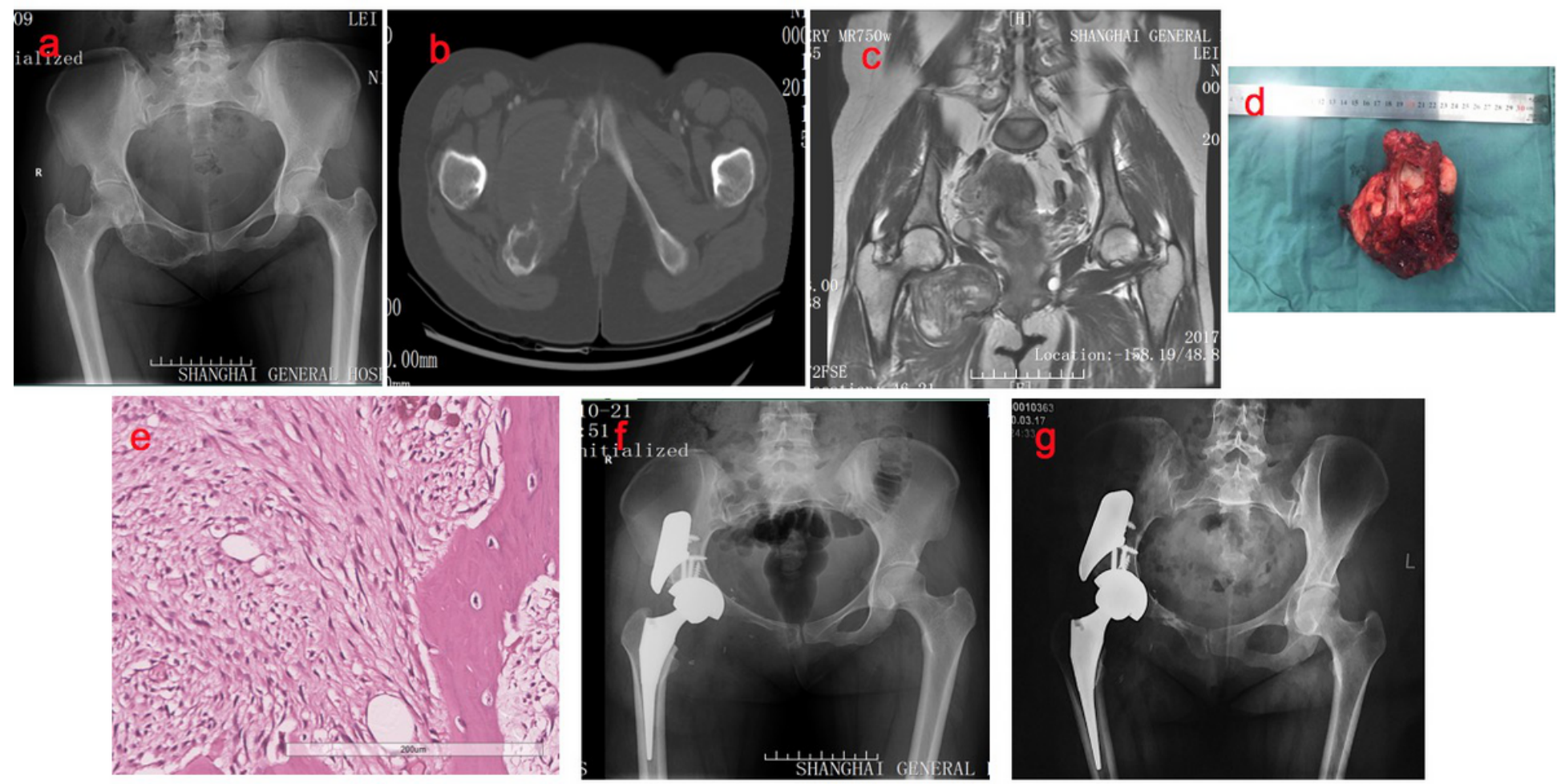

\section{Figure 4}

Female, 31 years old, pelvic osteosarcoma (a) Preoperative pelvic plain film showed expansive space occupying lesions involving ischium, pubis and acetabulum (b) Preoperative CT showed bone destruction of the right ischium and inferior pubic branch with soft tissue occupation (c) Preoperative MRI showed space occupying lesions in the right ischium, pubis and acetabulum (d) Intraoperative gross specimens (f) After operation, the bone and joint were in place and the right ischium was absent (g) Reexamination 2 years after operation showed that the joint was in place and the prosthesis was not loose. 


\section{Overall survival}

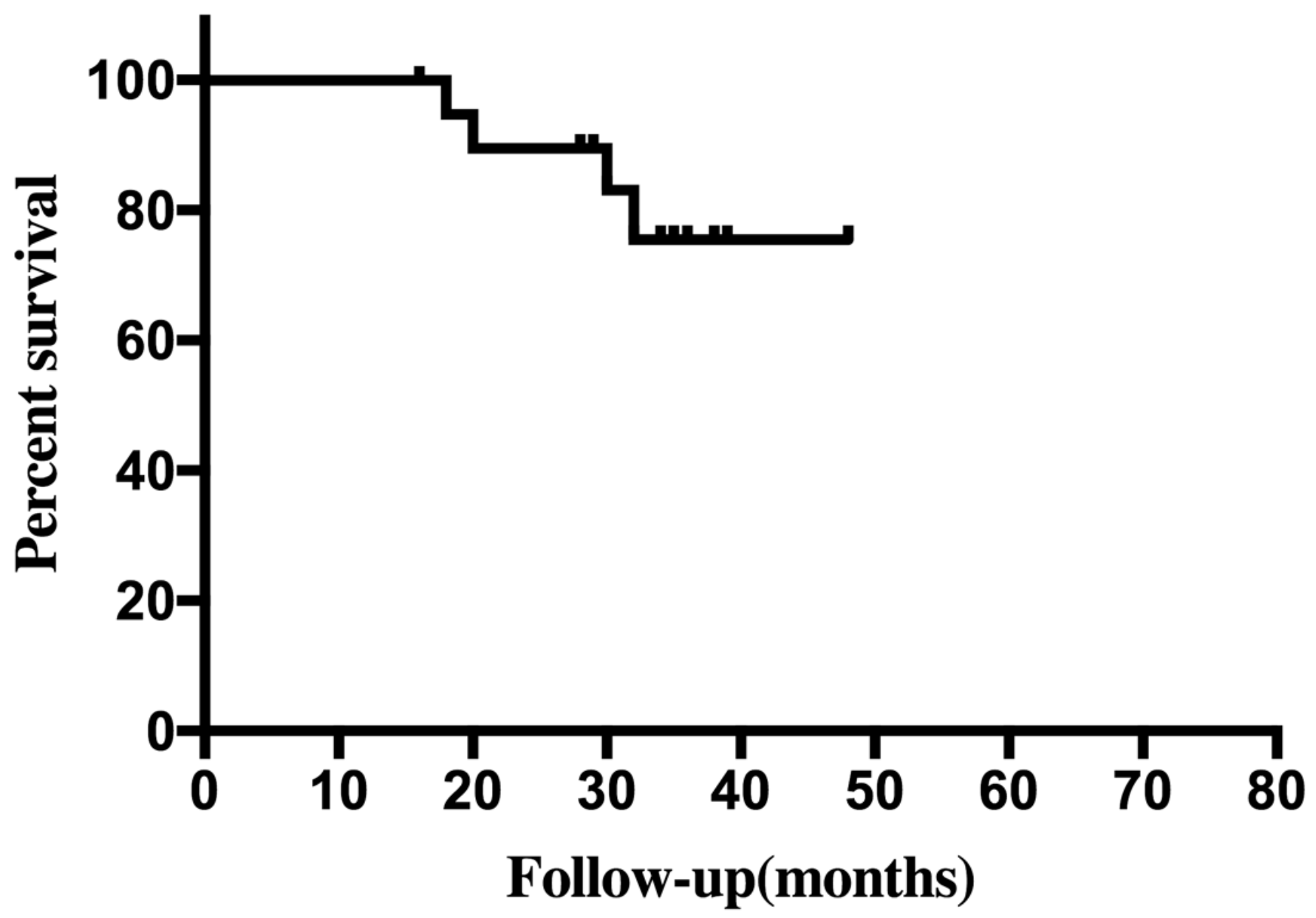

Figure 5

According to the Kaplan Meier survival curve, the three-year overall survival rate was $75.7 \%$. 